\title{
Comprehensive gene expression microarray analysis of Ets-1 blockade in PC3 prostate cancer cells and correlations with prostate cancer tissues: Insights into genes involved in the metastatic cascade
}

\author{
피미 \$ 9,' [\$ ' / ( 5, ANDREAS LINDSTROT, BERIT LANGER, \\ REINHARD BUETTNER and NICOLAS WERNERT
}

Institute of Pathology, University Hospital Bonn, D-53127 Bonn, Germany

Received November 16, 2010; Accepted January 7, 2011

DOI: $10.3892 / \mathrm{ijmm} .2011 .652$

\begin{abstract}
Ets-1 is the prototype of the ETS family of transcription factors and is suggested to play an important role in the malignant progression of prostatic carcinomas. Therefore, in this study we investigated the effect of blocking Ets-1 in PC3 prostate cancer cells on genes involved in the metastatic cascade, and correlated these findings with prostate cancer tissues. Two stable PC3 cell cultures were established by transfection with either an Ets-1 inverse antisense expression vector or a mock control vector. The effect of blocking Ets-1 on genes involved in the metastatic cascade was assessed by a comprehensive gene expression microarray analysis of Ets-1 inverse and mock control cells. Correlating the sets of genes found in the PC3 microarray data with prostate cancer tissues was performed by verifying the genes in a comprehensive gene expression microarray analysis of RNA extracted from laser microdissected normal prostate glands and from carcinoma glands taken from prostate cancer patients. Western blot analysis confirmed the presence of Ets-1 in mock cells and its absence in Ets-1 inverse cells. In the Ets-1 blockade microarray, many differentially expressed genes were found; however, only genes with a greater than 10-fold up- or downregulation between the Ets-1 blockade and mock control were considered significant. The genes were placed into four groups that play a role in the so-called metastatic cascade based on their known functions in proliferation, apoptosis, migration and angiogenesis. The genes found in the Ets-1 blockade microarray analysis were verified for their presence in the microarray analysis of prostate cancer tissues. Genes
\end{abstract}

Correspondence to: Professor Nicolas Wernert, Institute of Pathology, University Hospital Bonn, Sigmund-Freud-Str. 25, D-53127 Bonn, Germany

E-mail: nicolas.wernert@ukb.uni-bonn.de

Key words: proliferation, apoptosis, migration, angiogenesis, transcriptional regulation found in the microarray analysis of prostate cancer tissues with an $>2$-fold change and a $\mathrm{p}$-value $<0.01$ were considered significant. We identified sets of genes that are involved in the metastatic cascade and are known to be implicated in prostate cancer to show changes in the expression patterns due to the Ets-1 blockade in PC3 cells. Correlating these sets of genes with the findings in prostate cancer tissues, we identified 16 genes that are up- or down-regulated in healthy compared to tumor prostate glands. Further investigation revealed that 4 out of the 16 genes have been reported to be regulated by members of the ETS family. These findings provide in vitro and in vivo evidence for the importance of Ets-1 in the development and progression of prostate cancer.

\section{Introduction}

The development and progression of tumors involves different steps of the metastatic cascade, such as proliferation, apoptosis, migration, invasion and angiogenesis (1,2). A family of transcription factors called the ETS family is known to play important roles for such processes in both normal and neoplastic cells of different tissues (3-7).

The ETS family is characterized by an evolutionary highly conserved DNA-binding domain, termed the Ets domain, which consists of 80 amino acids with 4 tryptophan repeats (8). Several of the Ets genes are known to be rearranged to produce chimeric oncoproteins and are often found to be expressed in various types of human malignant tumors (9). In prostate cancer, unique chromosomal rearrangements are found in which the TMPRSS2 gene, encoding an androgen-regulated prostate-specific serine protease, is fused with several genes of the ETS family, most commonly ERG $(7,10,11)$. Such fusions increase the expression of rearranged Ets-factors as a response to androgens, resulting in an androgen-stimulated early stage prostate cancer progression (7).

Among these family members, Ets-1 is the prototype, which is expressed in various cell types and is reported to be involved in tumor progression by different mechanisms. Ets-1 is implicated in tumor angiogenesis and contributes to tumor proliferation and invasion by acting within both neoplastic 
cells and fibroblasts of the tumor stroma (12-18). It has been reported that Ets-1 is overexpressed in latent and clinically manifest prostatic carcinomas and that strong expression of Ets-1 is associated with poor tumor differentiation (14). In our previous studies, we reported that Ets-1 promotes proliferation, migration and invasion in various neoplastic cells such as melanoma, HeLa and glioma cells $(5,6,19)$.

Therefore, in this study we specifically blocked Ets-1 in PC3 prostate cancer cells and examined the effect of the blockade on genes involved in the metastatic cascade using a comprehensive gene expression microarray. Furthermore, we correlated our Ets-1 blockade gene expression microarray findings with the results of a second microarray analysis that we have performed on RNA extracted from laser microdissected healthy glands of the prostate proper (or the peripheral zone) and of prostate carcinomas.

We report that blocking Ets-1 in PC3 prostate cancer cells affects the expression of genes that may be involved in various aspects of the metastatic cascade, such as proliferation, apoptosis, migration and angiogenesis. Some of the genes affected are known to be involved in prostate cancer (20-38), while others have not yet been implicated. Correlating these genes with the findings of the microarray analysis of prostate cancer tissues, we identified a set of 16 genes that are up- or downregulated in healthy compared to cancerous prostate glands. Further analysis revealed that 4 out of the 16 genes have been reported to be regulated by members of the ETS family. Our present work provides in vivo and in vitro evidence for different roles of Ets-1 in prostate cancer.

\section{Materials and methods}

Cell culture. PC3 cells were grown in F-12K media with L-glutamine (Invitrogen, USA) supplemented with penicillin, streptomycin (Invitrogen) and 10\% heat-inactivated fetal calf serum (Invitrogen). In addition, G418 (4.3 mg/ml) (PAA, Austria) was added in the media of transfected cells.

Experimental blocking of Ets-1 in PC3 cells. Plasmids pcDNA3.1h-ets-1 inverse and pcDNA3.1(-) (mock control), respectively, were transfected into the PC 3 cells by the calcium phosphate method as previously described (39). At $24 \mathrm{~h}$ after transfection, selection was started using $4.3 \mathrm{mg} / \mathrm{ml} \mathrm{G} 418$ (PAA). The cells were then lysed by freezing and thawing at room temperature. A total of $20 \mu \mathrm{g}$ of protein was analyzed by $10 \%$ SDS-polyacrylamide gel electrophoresis and transferred onto nitrocellulose filters (Bio-Rad, USA). The filters were then blocked with 5\% dry milk in TBST $(50 \mathrm{mM}$ Tris, $150 \mathrm{mM}$ $\mathrm{NaCl}, 0.1 \%$ Tween-20, $\mathrm{pH} 7.5$ ) for $2 \mathrm{~h}$ at room temperature. The filters were then incubated in TBST and with anti-Ets-1 mouse monoclonal antibodies (1:5000; Transduction Laboratories), then with anti-mouse $\mathrm{IgG}$ horseradish peroxidase-conjugated secondary antibody (1:5000; Amersham Biosciences, USA). Antibodies were detected using ECL reagents (Amersham Biosciences). The housekeeping protein $\beta$-actin was used as an internal control and was detected using mouse monoclonal anti- $\beta$-actin antibody (1:5000; Sigma).

Microarray-analysis of mRNA expression of PC3 cells. RNA was isolated from cells with the RNeasy Mini kit (Qiagen,
Germany) as described by the manufacturer. The recommended DNase digestion was carried out with the RNase-free DNase set (Qiagen). The amount of isolated RNA was measured with the Nanodrop ND-1000 (Thermo Fisher Scientific, USA). The RNA was sent to Miltenyi Biotec (Germany), for microarray analysis and bioinformatical interpretation. The RNA was labelled with $\mathrm{Cy} 3$ and hybridized on the Whole Human Genome Oligo Microarray 4x44K (Agilent Technologies, USA) as described by the manufacturer. The microarray data were validated by qRT-PCR of a subset of genes.

Processing of human prostatectomy specimens. Fresh tissue samples from 5 moderately differentiated (Gleason-scores 6 and 7a), 4 poorly differentiated (Gleason-scores 8 and 9) prostate carcinomas as well as from 10 normal peripheral zones (prostate proper, sample sizes around $0.5 \times 0.5 \times 0.3 \mathrm{~cm}$ ) were taken immediately after radical prostatectomy and shock-frozen in liquid nitrogen with ice-cold isopentane. The age of the patients ranged from 45 to 83 (average 67.1) years. Frozen sections $(6 \mu \mathrm{m})$ were cut from the samples using a cryotome (Leica, Germany) and mounted on membrane-coated slides (MembraneSlides, $1 \mathrm{~mm}$ PEN) (Zeiss, Germany) for subsequent laser microdissection. One section was mounted on conventional slides and stained with hematoxylin and eosin (H\&E) for diagnostic evaluation by an experienced pathologist. Laser microdissection was carried out according to our previously published method (40-42). Frozen sections were dried for $2 \mathrm{~min}$ in the cryotome, washed for $2 \mathrm{~min}$ with $70 \%$ ethanol in DEPC-treated water and stained for $30 \mathrm{sec}$ in $1 \%$ Cresyl Violet diluted in 50\% ethanol-DEPC-treated water. Slides were then briefly washed in 70 and $100 \%$ ethanol, dried for $10 \mathrm{~min}$ and stored at $-80^{\circ} \mathrm{C}$ until use for laser microdissection.

Quality control. One frozen section from each sample was used for control of RNA integrity prior to laser-capture microdissection (LCM) using a Lasercapture Microscope (Axio Observer.Z1, Zeiss, Germany). The section was washed with $600 \mu \mathrm{l}$ DTT $(2 \mu \mathrm{M})$ in RLT buffer (RNeasy Mini kit, Qiagen) and vortexed for $30 \mathrm{sec}$. RNA-extraction was performed as described by the manufacturer. The recommended DNase digestion was carried out with an RNase-free DNase set (Qiagen). RNA integrity was measured with the Agilent Bioanalyzer 2100 (Agilent Technologies). Samples with a RIN factor $>6$ were used for LCM.

LCM. LCM of Cresyl Violet-stained sections was performed using the Axio Observer.Z1 Microscope (Zeiss) with the integrated Palm MicroBeam software (Zeiss). LCM was carried out under a 10x objective. Isolated normal or cancerous glands were collected in $200 \mu$ l AdhesiveCap tubes (Zeiss).

Microarray analysis of RNA from prostate samples. Total RNA was isolated from samples with the RNeasy Micro kit (Qiagen) as described by the manufacturer. The recommend DNase digestion was carried out with the RNase-free DNase set (Qiagen). The amount of the isolated RNA was determined using the Nanodrop ND-1000 (Thermo Fisher Scientific). Thereafter, an equal amount of RNA from normal peripheral glands on the one hand and from poorly and moderately differentiated prostate carcinoma glands on the other, were 
Table I. Cell proliferation genes up-regulated over 10-fold in Ets-1 blocked PC3 cells compared to mock control.

\begin{tabular}{ll}
\hline Gene & \multicolumn{1}{c}{ Full name } \\
\hline IGFBP3 & Insulin-like growth factor binding protein 3 \\
CD40 & CD40 molecule TNF receptor superfamily member 5 \\
FOXG1 & Forkhead box G1 \\
EPGN & Epithelial mitogen homolog (mouse) \\
STAT6 & Signal transducer and activator of transcription 6, interleukin-4 induced \\
ARNT2 & Aryl-hydrocarbon receptor nuclear translocator 2 \\
SPOCK1 & Sparc/osteonectin, cwcv and kazal-like domains proteoglycan (testican) 1 \\
FES & Feline sarcoma oncogene \\
RBP4 & Retinol binding protein 4, plasma \\
KDR & Kinase insert domain receptor (a type III receptor tyrosine kinase) \\
COL4A3 & Collagen, type IV, $\alpha$ 3 (Goodpasture antigen) \\
PLG & Plasminogen; lipoprotein, Lp(a)-like 2 pseudogene; solute carrier \\
& family 22 (extraneuronal monoamine transporter), member 3 \\
\hline
\end{tabular}

Table II. Cell proliferation genes down-regulated over 10-fold in Ets-1 blocked PC3 cells compared to mock control.

\begin{tabular}{ll}
\hline Gene & \\
\hline AR & Androgen receptor \\
IFI16 & Interferon, $\gamma$-inducible protein 16 \\
CCL2 & Chemokine (C-C motif) ligand 2 \\
DLC1 & Deleted in liver cancer 1; proline-rich nuclear receptor coactivator 2 \\
PRRX2 & Paired related homeobox 2 \\
GCG & Glucagon \\
PRKCQ & Protein kinase C, $\theta$ \\
P2RX7 & Purinergic receptor P2X, ligand-gated ion channel, 7 \\
EGR4 & Early growth response 4 \\
BST2 & Bone marrow stromal cell antigen 2 \\
PBX1 & Pre-B-cell leukemia homeobox 1 \\
FABP4 & Fatty acid binding protein 4, adipocyte \\
HCLS1 & Hematopoietic cell-specific Lyn substrate 1 \\
\hline
\end{tabular}

pooled to a final concentration of $300 \mathrm{ng}$ of RNA. These pools were sent to Miltenyi Biotec for microarray analysis and bioinformatical interpretation. The RNA was labelled with $\mathrm{Cy} 3$ and hybridized on the Whole Human Genome Oligo Microarray 4x44K (Agilent Technologies) according to the manufacturer's instructions. The microarray data were validated by qRT-PCR of a subset of genes.

Transcriptional Regulatory Element Database (TRED). We used the TRED database (43) in order to find out whether Ets-transcription factors are known to participate in the transcriptional regulation of 16 genes which we have found to be differentially expressed between PC3 cells with an Ets-1 blockade and PC3 mock control cells. For this purpose we entered the name of each gene in the TRED database and retrieved the list of reported regulators.

\section{Results}

Ets-1 can be experimentally blocked in PC3 cells. In order to experimentally block Ets-1, two stable PC3 cell cultures were established by transfection with either an Ets-1 inverse antisense expression vector or a mock control vector. Western blot analysis showed that PC3 cells expressing the mock control have detectable amounts of Ets-1, whereas PC3 cells expressing the Ets-1 inverse lacked detectable amounts of Ets-1 over a period of 121 days (44).

Gene expression analysis of Ets-1 blockade compared to mock control in PC3 cells using Whole Human Genome Oligo Microarrays. To study the effect of Ets-1 in PC3 prostate cancer cells upon expression of genes that are involved in the metastatic cascade, we analyzed the gene expression 
Table III. Apoptosis genes up-regulated over 10-fold in Ets-1 blocked PC3 cells compared to mock control.

\begin{tabular}{ll}
\hline Gene & \\
\hline IGFBP3 & Insulin-like growth factor binding protein 3 \\
BEX2 & Brain expressed X-linked 2 \\
ITGA1 & Integrin, $\alpha 1$ \\
NME5 & Non-metastatic cells 5, protein expressed in (nucleoside-diphosphate kinase) \\
VAV3 & Vav 3 guanine nucleotide exchange factor \\
BARHL1 & BarH-like homeobox 1 \\
ARNT2 & Aryl-hydrocarbon receptor nuclear translocator 2 \\
PYCARD & PYD and CARD domain containing \\
PTH & Parathyroid hormone \\
C5 & Complement component 5 \\
SNCA & Synuclein, $\alpha$ (non A4 component of amyloid precursor) \\
MMD2 & Monocyte to macrophage differentiation-associated 2 \\
PRKCE & Protein kinase C, $\varepsilon$ \\
COL4A3 & Collagen, type IV, $\alpha$ 3 (Goodpasture antigen) \\
PLG & Plasminogen; lipoprotein, Lp(a)-like 2 pseudogene; solute carrier \\
PARM1 & family 22 (extraneuronal monoamine transporter), member 3 \\
\hline
\end{tabular}

Table IV. Apoptosis genes down-regulated over 10-fold in Ets-1 blocked PC3 cells compared to mock control.

\begin{tabular}{ll}
\hline Gene & \\
\hline AR & Androgen receptor \\
IFI16 & Interferon, $\gamma$-inducible protein 16 \\
CCL2 & Chemokine $(C-C$ motif) ligand 2 \\
DLC1 & Deleted in liver cancer 1; proline-rich nuclear receptor coactivator 2 \\
FOXL2 & Forkhead box L2 \\
NLRP2 & NLR family, pyrin domain containing 2 \\
P2RX7 & Purinergic receptor P2X, ligand-gated ion channel, 7 \\
UNC5B & Unc-5 homolog B $(C$. elegans) \\
GAS2 & Growth arrest-specific 2; Fanconi anemia, complementation group F \\
XAF1 & XIAP associated factor 1 \\
LOC100137724 & Fanconi anemia, complementation group F; growth arrest-specific 2 \\
ENST00000367248 & Spectrin repeat containing, nuclear envelope 1 \\
TLR4 & Toll-like receptor 4 \\
KCNH8 & Potassium voltage-gated channel, subfamily H (eag-related), member 8 \\
\hline
\end{tabular}

profile in PC3 cells transfected with either an Ets-1 inverse antisense expression vector or a mock control vector. Genes whose expression was up- or down-regulated $>10$-fold, were considered significant and were selected for further analysis. A clustering analysis based on gene function placed different genes into four main processes: proliferation, apoptosis, migration and angiogenesis.

Genes involved in proliferation. Analysis of gene expression based on gene function identified genes with an $>10$-fold upor down-regulation that may be involved in cell proliferation (Tables I and II). Among these genes, AR, IGFBP3, CD40,
STAT6, SPOCK1, IFI16 and CCL2 have been reported to be involved in prostate cancer (20-27).

Genes involved in apoptosis. Genes with an $>10$-fold up- or down-regulation that may be involved in apoptosis are shown in Tables III and IV. Of these, AR, IGFBP3, VAV3, PTH, PRKCE, IFI16, CCL2, DLC1, UNC5B, GAS2, XAF1 and TLR4 have been reported to be involved in prostate cancer (20,24,28-35).

Genes involved in cell migration. Among the genes that were identified to be $>10$-fold up- or down-regulated and that may 
Table V. Cell migration genes up-regulated over 10-fold in Ets-1 blocked PC3 cells compared to mock control.

\begin{tabular}{ll}
\hline Gene & \\
\hline IGFBP3 & Insulin-like growth factor binding protein 3 \\
ITGA1 & Integrin, $\alpha 1$ \\
VAV3 & Vav 3 guanine nucleotide exchange factor \\
FOXG1 & Forkhead box G1 \\
OTX2 & Orthodenticle homeobox 2 \\
WNT5B & Wingless-type MMTV integration site family, member 5B \\
DCLK1 & Doublecortin-like kinase 1 \\
SPOCK1 & Sparc/osteonectin, cwcv and kazal-like domains proteoglycan (testican) 1 \\
RELN & Reelin \\
ISL1 & ISL LIM homeobox 1 \\
C5 & Complement component 5 \\
KDR & Kinase insert domain receptor (a type III receptor tyrosine kinase) \\
PLG & Plasminogen; lipoprotein, Lp(a)-like 2 pseudogene; solute carrier \\
& family 22 (extraneuronal monoamine transporter), member 3
\end{tabular}

Table VI. Cell migration genes down-regulated over 10-fold in Ets-1 blocked PC3 cells compared to mock control.

\begin{tabular}{ll}
\hline Gene & \multicolumn{1}{c}{ Full name } \\
\hline CCL2 & Chemokine (C-C motif) ligand 2 \\
DLC1 & Deleted in liver cancer 1; proline-rich nuclear receptor coactivator 2 \\
AMOT & Angiomotin \\
UNC5B & Unc-5 homolog B $(C$. elegans $)$ \\
DRD5 & Dopamine receptor D5 \\
TIE1 & Tyrosine kinase with immunoglobulin-like and EGF-like domains 1 \\
MTSS1 & Metastasis suppressor 1 \\
\hline
\end{tabular}

be involved in cell migration, IGFBP3, VAV3, SPOCK1, CCL2, DLC1 and MTSS1 are known to be implicated in prostate cancer $(22-24,27-29,32,33,36)$ (Tables V and VI).

Genes involved in angiogenesis. Genes that may be involved in angiogenesis and were found to be $>10$-fold up or downregulated are listed in Tables VII and VIII. Among these genes, VAV3, KDR and TIE1 are known to be implicated in prostate cancer $(32,33,37,38)$.

Genes identified in prostate cancer tissues by Whole Human Genome Oligo Microarray. The genes from Tables I-VIII that we found to be up- or down-regulated in the gene expression microarray analysis of poorly and moderately differentiated human prostate cancers compared to healthy glands of the peripheral zone with a fold-change of $>2$ and a p-value $<0.01$ are summarized in Tables IX and X. Among these genes, CD40, IGFBP3, FES and TLR4 are reported to be regulated by members of the ETS family according to the TRED database. Furthermore, we found Ets-1 to be up-regulated 2.6-fold in prostate carcinomas compared to healthy glands. Previous studies have also shown that Ets-1 is overexpressed in latent and clinically manifest prostatic carcinomas and strong expression of Ets-1 is associated with poor tumor differentiation (14).

\section{Discussion}

Tumor development and progression is characterized by changes in gene expression patterns involving various processes responsible for different steps of the so-called metastatic cascade (such as proliferation, apoptosis, migration and angiogenesis) (1,2). In our previous studies, we reported that the Ets-1 transcription factor plays a role in various of these steps such as proliferation, migration and invasion in different neoplastic cells including melanoma, HeLa and glioma cells $(5,6,19)$.

In this study, we report that blocking Ets-1 in PC3 prostate cancer cells likewise affects the expression of genes that may be involved in the metastatic cascade. In a recent study, we have examined the effect of blocking Ets-1 in PC3 cells, and we have found that Ets-1 promotes cell migration, but not invasion (44), while others have shown that inhibition of Ets-1 blocks cell proliferation (45). Furthermore, we have correlated these microarray findings with those of microarray analysis of prostate cancer tissues. Several of the genes affected are 
Table VII. Angiogenesis genes up-regulated over 10-fold in Ets-1 blocked PC3 cells compared to mock control.

\begin{tabular}{ll}
\hline Gene & \multicolumn{1}{c}{ Full name } \\
\hline ITGA1 & Integrin, $\alpha 1$ \\
VAV3 & Vav 3 guanine nucleotide exchange factor \\
EPGN & Epithelial mitogen homolog (mouse) \\
EMCN & Endomucin \\
BAI3 & Brain-specific angiogenesis inhibitor 3 \\
NPY1R & Neuropeptide Y receptor Y1 \\
GUCY1A3 & Guanylate cyclase 1, soluble, $\alpha 3$ \\
KDR & Kinase insert domain receptor (a type III receptor tyrosine kinase) \\
COL4A3 & Collagen, type IV, $\alpha$ 3 (Goodpasture antigen) \\
PLG & Plasminogen; lipoprotein, Lp(a)-like 2 pseudogene; solute carrier \\
& family 22 (extraneuronal monoamine transporter), member 3 \\
\hline
\end{tabular}

Table VIII. Angiogenesis genes down-regulated over 10-fold in Ets-1 blocked PC3 cells compared to mock control.

Gene

Full name

\begin{tabular}{ll}
\hline PRRX2 & Paired related homeobox 2 \\
PRKCQ & Protein kinase C, $\theta$ \\
AMOT & Angiomotin \\
DRD5 & Dopamine receptor D5 \\
TIE1 & Tyrosine kinase with immunoglobulin-like and EGF-like domains 1 \\
ADRA2B & Adrenergic, $\alpha-2 \mathrm{~B}-$, receptor \\
\hline
\end{tabular}

already known to be involved in prostate cancer, while others have not yet been implicated.

Cell proliferation. Among the genes regulated by Ets-1 in PC3 cells and involved in cell proliferation, we identified AR, IFI16, CCL2, IGFBP3, CD40, STAT6, and SPOCK1 which are known to be implicated in prostate cancer (20-27) (Tables I and II).

AR, IFI16 and CCL2 were found to be more than 10-fold down-regulated in Ets-1 blocked PC3 cells compared to mock control cells, whereas IGFBP3, CD40, EPGN, STAT6 and SPOCK1 were found to be more than a 10-fold up-regulated. This means that Ets-1 induces the gene expression of AR, IFI16 and CCL2. In contrast, Ets-1 inhibits the expression of IGFBP3, CD40, EPGN, STAT6 and SPOCK1.

Briefly, among the genes that are induced by Ets- 1 in PC3 cells and known to be involved in prostate cancer are the androgen receptor (AR), IFI16 and CCL2. Both normal and neoplastic growth of the prostate gland is dependent on the expression and function of the AR (20). In prostate cancer, the activity of the AR is critical for cell proliferation and progression of the disease $(46,47)$. Increased expression of IFI16 in normal human prostate epithelial cells is associated with cellular senescence-associated cell growth arrest, whereas transcriptional silencing of IFI16 in such cells contributes to the development of prostate cancer (21). It has been suggested that CCL2 promotes PC3 prostate cancer cell survival (22), as well as prostate cancer tumorigenesis and metastasis (23).
On the other hand, genes that are inhibited by Ets-1 in PC3 cells and known to be involved in prostate cancer include IGFBP3, CD40, STAT6 and SPOCK1. Briefly, IGFBP3 has been shown to inhibit proliferation, adhesion, migration, and invasion processes of prostate tumor cells (24). Studies that investigated CD40 expression in tissues from patients with prostate cancer have concluded that invasive prostate cancer is a CD40-negative tumor (25). STAT6 has been suggested to be a survival factor in prostate cancer and to be involved in prostate cancer progression (26). Finally, SPOCK1 has been reported to be up-regulated in prostate cancer (27).

Apoptosis. Among the genes affected by Ets-1 in PC3 cells and involved in apoptosis, we analyzed AR, IGFBP3, VAV3, PTH, PRKCE, IFI16, CCL2, DLC1, XAF1 and TLR4 which are known to be involved in prostate cancer $(20,24,28-35)$ (Tables III and IV). AR, IFI16, CCL2, DLC1, XAF1 and TLR4 were found to be more than 10 -fold down-regulated in Ets-1 blocked PC3 cells compared to mock control cells, whereas IGFBP3, VAV3, PRKCE, and PTH were shown to be more than a 10 -fold up-regulated. This demonstrates that Ets-1 induces the gene expression of AR, IFI16, CCL2, DLC1, XAF1 and TLR4. In contrast, Ets-1 inhibits the expression of the IGFBP3, VAV3, PRKCE, and PTH genes.

Briefly, among the genes that are induced by Ets-1 in PC3 cells and are known to be involved in prostate cancer are the AR, IFI16, CCL2, DLC1, XAF1 and TLR4. The involvement 
Table IX. Genes from Tables I-VIII that were found to be down-regulated in human prostate carcinomas compared to healthy glands of the peripheral zone.

\begin{tabular}{ll}
\hline Gene & \\
\hline VAV3 & Vav 3 guanine nucleotide exchange factor \\
FES & Feline sarcoma oncogene \\
PYCARD & PYD and CARD domain containing \\
TLR4 & Toll-like receptor 4 \\
IGFBP3 & Insulin-like growth factor binding protein 3 \\
CD40 & CD40 molecule, TNF receptor superfamily member 5 \\
GAS2 & Growth arrest-specific 2; Fanconi anemia, complementation group F \\
SNCA & Synuclein, $\alpha$ (non A4 component of amyloid precursor) \\
AMOT & Angiomotin \\
NPY1R & Neuropeptide Y receptor Y1 \\
\hline
\end{tabular}

Candidate genes with a fold-change of $>2$ and a p-value $<0.01$ are summarized in this Table.

Table X. Genes from Tables I-VIII that were found to be upregulated in human prostate carcinomas compared to healthy glands of the peripheral zone.

\begin{tabular}{ll} 
Gene & \multicolumn{1}{c}{ Full name } \\
\hline PRRX2 & Paired related homeobox 2 \\
ISL1 & ISL LIM homeobox 1 \\
NLRP2 & NLR family, pyrin domain containing 2 \\
BST2 & Bone marrow stromal cell antigen 2 \\
FOXL2 & Forkhead box L2 \\
EGR4 & Early growth response 4 \\
\hline
\end{tabular}

Candidate genes with a fold-change of $>2$ and a p-value $<0.01$ are summarized in this Table.

of the AR, IFI16 and CCL2 in prostate cancer has been discussed above. Studies have indicated that down-regulation of DLC-1 is associated with a variety of cancer types including prostate cancer $(28,29)$. XAF1 has been suggested to contribute to prostate cancer pathogenesis by disrupting the balance of the apoptosis machinery (30). TLR4 is implicated in tumor cell invasion, survival, and metastasis in a variety of cancers $(31,48,49)$. Furthermore, knockdown of TLR4 in PC3 cells has been reported to result in a reduction of cell migration and invasion (31).

On the other hand, genes that are inhibited by Ets-1 in PC3 cells and known to be implicated in prostate cancer include IGFBP3, VAV3, PRKCE, and PTH. The role of IGFBP3 in prostate cancer has been discussed above. VAV3 has been shown to be overexpressed in human prostate cancer, to activate the AR and to stimulate the growth of prostate cancer cells $(32,33)$. PRKCE has been implicated in the promotion of human prostate cancer cell survival (34). Increased PTH concentrations have been detected in patients with advanced prostate cancer (35).
Cell migration. In a set of genes regulated by Ets-1 in PC3 cells and involved in cell migration, we analyzed IGFBP3, VAV3, SPOCK1, CCL2, DLC1 and MTSS1 which are known to be implicated in prostate cancer $(22-24,27-29,32,33,36)$ (Tables V and VI). CCL2, DLC1 and MTSS1 are shown to be more than a 10-fold down-regulated in Ets-1 blocked PC3 cells compared to mock control cells, whereas IGFBP3, VAV3 and SPOCK 1 are shown to be more than 10-fold up-regulated. This shows that Ets-1 induces the gene expression of CCL2, DLC1 and MTSS1. In contrast, Ets-1 inhibits the expression of the IGFBP3, VAV3 and SPOCK1 genes.

Briefly, the roles of CCL2 and DLC1 which are induced by Ets-1 in PC3 cells and shown to be more than 10-fold down-regulated have been discussed. It has been reported that reduction of MTSS1 gene expression in prostate cancer may contribute to tumor growth, development, and metastasis (36). On the other hand, genes that are inhibited by Ets-1 in PC3 cells and have more than 10-fold up-regulation such as IGFBP3, VAV3 and SPOCK1 have been discussed above.

Angiogenesis. Genes involved in angiogenesis with more than a 10-fold up- and down-regulation due to blocking of Ets-1 in PC3 cells with known roles in prostate cancer include VAV3, KDR and TIE1 $(32,33,37,38)$ (Tables VII and VIII).

Briefly, TIE1 is down-regulated, whereas VAV3 and KDR are up-regulated after Ets-1 blockade. This means that Ets-1 induces the expression of the TIE1 gene, and in contrast, inhibits the expression of the VAV3 and KDR genes. TIE1 has been found to be expressed at a higher level in cancer patients compared to healthy donors (37). The role of the up-regulated gene KDR in prostate cancer has been discussed above. Finally, KDR has been shown to be expressed highly in prostate adenocarcinomas (38).

Correlations with prostate cancer tissues. The identified sets of genes shown above were verified for their presence in the microarray analysis of laser microdissected normal peripheral zone glands and a series of human prostate carcinomas. A set of 16 genes was found to be up- or down-regulated in prostate 
carcinomas compared to healthy glands of the peripheral zone with an $>2$-fold-change and a $\mathrm{p}<0.01$ (Tables IX and $\mathrm{X})$. Among these 16 genes, CD40, IGFBP3, FES and TLR4 were found to be down-regulated in tumor tissues (Table IX) and reported to be regulated by members of the ETS family SPI-1, FLI-1, SPI-1 and SPI-1/ETS-1, respectively (50-53). Furthermore, we found Ets-1 to be up-regulated 2.6-fold in prostate carcinomas compared to healthy glands.

It is worth noting that CD40, IGFBP3 and FES which were reported to be regulated by ETS family members (43), were found to be up-regulated in the PC3 Ets-1 blockade, while down-regulated in the tumor prostate tissues along with Ets-1 up-regulation. These observations support a possible role of Ets-1 in regulating these genes in prostate cancer and may provide more insights into events of the metastatic cascade.

In conclusion, our present work provides in vitro and in vivo evidence for roles of the Ets-1 transcription factor in the biology of prostate cancer. It shows changes in gene expression patterns due to Ets-1 blockade in PC3 cells, as well as correlations with human prostate cancer tissues. These patterns may involve various steps of the metastatic cascade (such as proliferation, apoptosis, migration and angiogenesis). The findings seem important with respect to frequent translocations of different ETS family members in prostate carcinoma $(7,10,11)$, since Ets- 1 is the prototype of this family. Thus, the differentially expressed genes and pathways provide a more comprehensive view into the broad roles that Ets-1 might play in the development and progression of prostate cancer.

Additional studies such as DNA binding- and co-transfection assays are needed in order to gain additional knowledge about the exact molecular mechanisms by which Ets-1 regulates the expression of these sets of genes. Particular candidates may prove useful as diagnostic or prognostic markers or even novel therapeutic targets.

\section{Acknowledgements}

We are grateful to the DFG (Deutsche Forschungsgemeinschaft; German Research Association, grant no. WE 1104/11-1) and the Deutsche Krebshilfe (German Cancer Aid, grant no. 107827) for financial support.

\section{References}

1. Kohn EC: Invasion and metastasis: biology and clinical potential. Pharmacol Ther 52: 235-244, 1991.

2. Duffy MJ, McGowan PM and Gallagher WM: Cancer invasion and metastasis: changing views. J Pathol 214: 283-293, 2008.

3. Oikawa T: ETS transcription factors: possible targets for cancer therapy. Cancer Sci 95: 626-633, 2004.

4. Hashiya N, Jo N, Aoki M, et al: In vivo evidence of angiogenesis induced by transcription factor Ets-1: Ets-1 is located upstream of angiogenesis cascade. Circulation 109: 3035-3041, 2004.

5. Rothhammer T, Hahne JC, Florin A, et al: The Ets-1 transcription factor is involved in the development and invasion of malignant melanoma. Cell Mol Life Sci 61: 118-128, 2004.

6. Hahne JC, Okuducu AF, Kaminski A, Florin A, Soncin F and Wernert N: Ets-1 expression promotes epithelial cell transformation by inducing migration, invasion and anchorage-independent growth. Oncogene 24: 5384-5388, 2005.

7. He J, Pan Y, Hu J, Albarracin C, Wu Y and Dai JL: Profile of Ets gene expression in human breast carcinoma. Cancer Biol Ther 6: 76-82, 2007.
8. Wasylyk B, Hahn SL and Giovane A: The Ets family of transcription factors. Eur J Biochem 211: 7-18, 1993.

9. Hahne JC, Okuducu AF, Sahin A, Fafeur V, Kiriakidis S and Wernert N: The transcription factor ETS-1: its role in tumor development and strategies for its inhibition. Mini Rev Med Chem 8: 1095-1105, 2008.

10. Tomlins SA, Rhodes DR, Perner S, et al: Recurrent fusion of TMPRSS2 and ETS transcription factor genes in prostate cancer. Science 310: 644-648, 2005.

11. Furusato B, Tan SH, Young D, et al: ERG oncoprotein expression in prostate cancer: clonal progression of ERG-positive tumor cells and potential for ERG-based stratification. Prostate Cancer Prostatic Dis 13: 228-237, 2010.

12. Behrens P, Rothe M, Wellmann A, Krischler J and Wernert N: The Ets-1 transcription factor is up-regulated together with MMP 1 and MMP 9 in the stroma of pre-invasive breast cancer. J Pathol 194: 43-50, 2001.

13. Behrens P, Mathiak M, Mangold E, et al: Stromal expression of invasion-promoting, matrix-degrading proteases MMP-1 and -9 and the Ets 1 transcription factor in HNPCC carcinomas and sporadic colorectal cancers. Int J Cancer 107: 183-188, 2003.

14. Alipov G, Nakayama T, Ito M, et al: Overexpression of Ets-1 proto-oncogene in latent and clinical prostatic carcinomas. Histopathology 46: 202-208, 2005.

15. Hahne JC, Kummer S, Heukamp LC, et al: Regulation of protein tyrosine kinases in tumor cells by the transcription factor Ets-1. Int J Oncol 35: 989-996, 2009.

16. Wernert N, Stanjek A, Kiriakidis S, et al: Inhibition of angiogenesis in vivo by ets-1 antisense oligonucleotides-inhibition of Ets-1 transcription factor expression by the antibiotic fumagillin. Angew Chem Int Ed Engl 38: 3228-3231, 1999.

17. Wernert N, Gilles F, Fafeur V, et al: Stromal expression of c-Ets1 transcription factor correlates with tumor invasion. Cancer Res 54: 5683-5688, 1994.

18. Wernert N, Raes MB, Lassalle P, et al: c-ets1 proto-oncogene is a transcription factor expressed in endothelial cells during tumor vascularization and other forms of angiogenesis in humans. Am J Pathol 140: 119-127, 1992.

19. Sahin A, Vercamer C, Kaminski A, et al: Dominant-negative inhibition of Ets 1 suppresses tumor growth, invasion and migration in rat C6 glioma cells and reveals differentially expressed Ets 1 target genes. Int J Oncol 34: 377-389, 2009.

20. Koochekpour S: Androgen receptor signaling and mutations in prostate cancer. Asian J Androl 12: 639-657, 2010.

21. Alimirah F, Chen J, Davis FJ and Choubey D: IFI16 in human prostate cancer. Mol Cancer Res 5: 251-259, 2007.

22. Roca H, Varsos Z and Pienta KJ: CCL2 protects prostate cancer PC3 cells from autophagic death via phosphatidylinositol 3-kinase/AKT-dependent survivin up-regulation. J Biol Chem 283: 25057-25073, 2008.

23. Zhang J, Patel L and Pienta KJ: CC chemokine ligand 2 (CCL2) promotes prostate cancer tumorigenesis and metastasis. Cytokine Growth Factor Rev 21: 41-48, 2009.

24. Massoner P, Colleselli D, Matscheski A, et al: Novel mechanism of IGF-binding protein-3 action on prostate cancer cells: inhibition of proliferation, adhesion, and motility. Endocr Relat Cancer 16: 795-808, 2009.

25. Palmer DH, Hussain SA, Ganesan R, et al: CD40 expression in prostate cancer: a potential diagnostic and therapeutic molecule. Oncol Rep 12: 679-682, 2004.

26. Das S, Roth CP, Wasson LM and Vishwanatha JK: Signal transducer and activator of transcription-6 (STAT6) is a constitutively expressed survival factor in human prostate cancer. Prostate 67: 1550-1564, 2007.

27. Wlazlinski A, Engers R, Hoffmann MJ, et al: Downregulation of several fibulin genes in prostate cancer. Prostate 67: 1770-1780, 2007.

28. Liao YC and Lo SH: Deleted in liver cancer-1 (DLC-1): a tumor suppressor not just for liver. Int J Biochem Cell Biol 40: 843-847, 2008.

29. Zhou X, Yang XY and Popescu NC: Synergistic antineoplastic effect of DLC1 tumor suppressor protein and histone deacetylase inhibitor, suberoylanilide hydroxamic acid (SAHA), on prostate and liver cancer cells: perspectives for therapeutics. Int J Oncol 36: 999-1005, 2010.

30. Fang X, Liu Z, Fan Y, et al: Switch to full-length of XAF1 mRNA expression in prostate cancer cells by the DNA methylation inhibitor. Int J Cancer 118: 2485-2489, 2006. 
31. Hua D, Liu MY, Cheng ZD, et al: Small interfering RNA-directed targeting of Toll-like receptor 4 inhibits human prostate cancer cell invasion, survival, and tumorigenicity. Mol Immunol 46: 2876-2884, 2009.

32. Dong Z, Liu Y, Lu S, et al: Vav3 oncogene is overexpressed and regulates cell growth and androgen receptor activity in human prostate cancer. Mol Endocrinol 20: 2315-2325, 2006.

33. Lee K, Liu Y, Mo JQ, Zhang J, Dong Z and Lu S: Vav3 oncogene activates estrogen receptor and its overexpression may be involved in human breast cancer. BMC Cancer 8: 158, 2008.

34. McJilton MA, Van Sikes C, Wescott GG, et al: Protein kinase $\mathrm{C}$ epsilon interacts with Bax and promotes survival of human prostate cancer cells. Oncogene 22: 7958-7968, 2003

35. Quirosa Flores S, Varsavsky M, Valle Diaz De La Guardia F, et al: Secondary hyperparathyroidism in advanced prostate cancer. Endocrinol Nutr 57: 100-104, 2010.

36. Loberg RD, Neeley CK, Adam-Day LL, et al: Differential expression analysis of MIM (MTSS1) splice variants and a functional role of MIM in prostate cancer cell biology. Int J Oncol 26 : $1699-1705,2005$

37. Smirnov DA, Foulk BW, Doyle GV, Connelly MC, Terstappen LW and O'Hara SM: Global gene expression profiling of circulating endothelial cells in patients with metastatic carcinomas. Cancer Res 66: 2918-2922, 2006.

38. Bai AS, Zeng H, Li X, Wei Q, Li H and Yang YR: Expression of kinase insert domain-containing receptor in prostate adenocarcinoma. Zhonghua Nan Ke Xue 13: 324-326, 2007 (In Chinese).

39. Chen CA and Okayama H: Calcium phosphate-mediated gene transfer: a highly efficient transfection system for stably transforming cells with plasmid DNA. Biotechniques 6: 632-638, 1988.

40. Wellmann A, Wollscheid V, Lu H, et al: Analysis of microdissected prostate tissue with ProteinChip arrays - a way to new insights into carcinogenesis and to diagnostic tools. Int $\mathbf{J}$ Mol Med 9: 341-347, 2002.

41. Okuducu AF, Janzen V, Hahne JC, Ko Y and Wernert N: Influence of histochemical stains on quantitative gene expression analysis after laser-assisted microdissection. Int $\mathrm{J}$ Mol Med 11: 449-453, 2003

42. Okuducu AF, Hahne JC, Von Deimling A and Wernert N: Laser-assisted microdissection, techniques and applications in pathology (Review). Int J Mol Med 15: 763-769, 2005.
43. Transcriptional Regulatory Element Database, http://rulai.cshl. edu/TRED/, Cold Spring Harbor Laboratory, Cold Spring, Harbor, NY.

44. \$ COOI', Langer B, Lindstrot A, etlal: Ets-1 is impliFDMCDIQUMH regulation of androgen Co-regulator FHL2 and reveals specificity for migration, but not invasion, of PC3 Prostate Cancer Cells. Oncol Rep (In Press).

45. Holterman CE, Franovic A, Payette J and Lee S: ETS-1 oncogenic activity mediated by transforming growth factor alpha. Cancer Res 70: 730-740, 2010.

46. Feldman BJ and Feldman D: The development of androgenindependent prostate cancer. Nat Rev Cancer 1: 34-45, 2001.

47. Balk SP: Androgen receptor as a target in androgen-independent prostate cancer. Urology 60: 132-139, 2002.

48. Hiratsuka S, Watanabe A, Sakurai Y, et al: The S100A8-serum amyloid A3-TLR4 paracrine cascade establishes a pre-metastatic phase. Nat Cell Biol 10: 1349-1355, 2008.

49. Andreani V, Gatti G, Simonella L, Rivero V and Maccioni M: Activation of Toll-like receptor 4 on tumor cells in vitro inhibits subsequent tumor growth in vivo. Cancer Res 67: 10519-10527, 2007.

50. Nguyen VT and Benveniste EN: Involvement of STAT-1 and ets family members in interferon-gamma induction of CD40 transcription in microglia/macrophages. J Biol Chem 275: 23674-23684, 2000.

51. Prieur A, Tirode F, Cohen P and Delattre O: EWS/FLI-1 silencing and gene profiling of Ewing cells reveal downstream oncogenic pathways and a crucial role for repression of insulin-like growth factor binding protein 3. Mol Cell Biol 24: 7275-7283, 2004.

52. Heydemann A, Juang G, Hennessy K, Parmacek MS and Simon MC: The myeloid-cell-specific c-fes promoter is regulated by Sp1, PU.1, and a novel transcription factor. Mol Cell Biol 16: 1676-1686, 1996.

53. Rehli M, Poltorak A, Schwarzfischer L, Krause SW, Andreesen R and Beutler B: PU.1 and interferon consensus sequence-binding protein regulate the myeloid expression of the human Toll-like receptor 4 gene. J Biol Chem 275: 9773-9781, 2000. 